revised 2016 World Health Organization diagnostic criteria. Blood Res 2019;54:284-5.

2. GBD 2016 Lifetime Risk of Stroke Collaborators. Global, regional, and country-specific lifetime risks of stroke, 1990 and 2016. N Engl J Med 2018;379:2429-37.

3. Economides MP, Verstovsek S, Pemmaraju N. Novel therapies in myeloproliferative neoplasms (MPN): beyond JAK inhibitors. Curr Hematol Malig Rep 2019;14:460-8.

4. Anderson N, Bonsell K. Ischemic stroke as the presenting symptom in a young patient with essential thrombocythemia caused by CALR gene mutation. Neurology (AAN 67th Annual Meeting Abstracts) 2015;84(Suppl):abst P2.253.

5. Beer PA, Campbell PJ, Scott LM, et al. MPL mutations in myeloproliferative disorders: analysis of the PT-1 cohort. Blood 2008; 112:141-9.

6. Pietra D, Li S, Brisci A, et al. Somatic mutations of JAK2 exon 12 in patients with JAK2 (V617F)-negative myeloproliferative disorders. Blood 2008;111:1686-9.

7. Stefanou MI, Richter H, Härtig F, et al. Recurrent ischaemic cerebrovascular events as presenting manifestations of myeloproliferative neoplasms. Eur J Neurol 2019;26:903-e64.

\section{Response to the letter by Langabeer on "Retrospective screening for Philadelphia-negative myeloproliferative neoplasms in patients with cerebral infarctions as revealed using the revised 2016 World Health Organization diagnostic criteria"}

TO Dr.LANGABEER: Thank you for your interests in our observations and good suggestions. Thromboembolism can precede overt presentation of a myeloproliferative neoplasm (MPN) by a few years. In this context, your suggestions on the molecular testing in stroke patients may be reasonable. Mayo Clinic has screened JAK2V617F in patients with 664 patients with non-splanchnic thrombosis, including 136 stokes. JAKV617F was found in only 6 patients, and the mutant allele burden was low in all instances (2.27.5\%). Based on these observations, they concluded that mutation screening was not warranted as part of the hypercoagulable work-up in the absence of MPN [1]. In addition,
$J A K 2 V 617 F$ seems to be found in normal population [2]. Furthermore, currently early detection of MPN driver gene mutations does not confer therapeutic implications in stroke patients with normal hematologic features, because available hematologic intervention such as cytoreductive therapy is indicated only in patients with overt thrombocytosis or erythrocytosis. Accordingly, we need to pay more attention to hematologic alterations at the time of diagnosis and regular follow-up of hematologic tests in patients with stroke rather than routine molecular screening, at least in daily clinical practice. For the selected cases of embolic stoke or cerebrovascular sinus thrombosis with otherwise undetermined source, molecular screening for MPN may be reasonable even in the absence of abnormal hematologic findings [3]. Collectively, molecular screening for MPN in stoke patients is still an open question, requiring prospective investigations and discussions.

Ik-Chan Song

Division of Hematology/Oncology, Department of Internal Medicine, College of Medicine, Chungnam National University, Daejeon, Korea

Correspondence to: Ik-Chan Song Division of Hematology/Oncology, Department of Internal Medicine, College of Medicine, Chungnam National University, 266 Munhwa-ro, Daejeon 35015, Korea E-mail: petrosong@naver.com

Received on Feb. 17, 2020; Accepted on Mar. 7, 2020 https://doi.org/10.5045/br.2020.55.1.68

\section{Authors' Disclosures of Potential Conflicts of Interest}

No potential conflicts of interest relevant to this article were reported.

\section{REFERENCES}

1. Pardanani A, Lasho TL, Hussein K, et al. JAK2V617F mutation screening as part of the hypercoagulable work-up in the absence of splanchnic venous thrombosis or overt myeloproliferative neoplasm: assessment of value in a series of 664 consecutive patients. Mayo Clin Proc 2008;83:457-9.

2. Xu X, Zhang Q Luo J, et al. JAK2(V617F): Prevalence in a large Chinese hospital population. Blood 2007;109:339-42.

3. Trifan G, Shafi N, Testai FD. Implications of janus kinase 2 mutation in embolic stroke of unknown source. J Stroke Cerebrovasc Dis 2018;27:2572-8. 